\title{
A Low-power Portable Digital Spectroscopy for CZT Gamma-ray Detector
}

\author{
Qibin Zheng ${ }^{1,2, a}$, Changqing Feng ${ }^{1,2, b^{*}}$, Hang Su ${ }^{1,2}$, Danyang Zhu ${ }^{1,2}$, Shubin \\ $\mathrm{Liu}^{1,2}$ and Qi An ${ }^{1,2}$ \\ ${ }^{1}$ Modern Physics Department, University of Science and Technology of China \\ ${ }^{2}$ The State Key Laboratory of Particle Detection and Electronics, USTC \\ aqbzheng@mail.ustc.edu.cn, bfengcq@ustc.edu.cn
}

Keywords: Spectroscopy, CdZnTe (CZT), Universal Serial Bus (USB), Portable, Low-power, Field Programmable Gate Array (FPGA).

\begin{abstract}
A portable, low power, digital spectroscopy for CdZnTe (CZT) detector is designed and described in this paper. Its readout electronics is implemented with waveform sampling, Flash-based FPGA (Field Programmable Gate Array) and USB (universal serial bus) technique. The CZT detector, with an effective thickness of $3 \mathrm{~mm}$ and a diameter of $5 \mathrm{~mm}$, is mounted on the same PCB board with high voltage supply module and low noise preamplifier. Current signal from CZT detector is integrated by a charge sensitive amplifier and then shaped by a CR-RC ${ }^{3}$ shaper. The shaped signal is sampled by a 12-bit ADC and then processed by an IGLOO Flash-based low power FPGA in a digital module. A USB interface is adopted both for low voltage and high voltage power supply, and communicating with a host computer, which results in a portable solution with maximum power consumption of about $450 \mathrm{~mW}$. In the host computer, a graphic user interface (GUI) software is developed based on Labwindows/CVI, for real-timely displaying the energy spectrum or waveform of shaping signal. Test results showed that the dynamic range of the spectroscopy can reach 240fC (equivalent to about $7 \mathrm{MeV}$ energy deposition in CZT detector) and the intrinsic noise (in RMS) for the electronics is less than $0.3 \mathrm{fC}$ (equivalent to about $8.7 \mathrm{keV}$ ).
\end{abstract}

\section{Introduction}

CZT (CdZnTe) is an excellent material for high efficiency, high-resolution nuclear radiation detectors in the area of environmental monitoring, radiation medicine, industrial non-destructive testing, home-land security, etc. It is widely applied because of its high performance on X-ray and $\gamma$-ray detection and benefits from large band-gap energy which allows us to operate the detector on room temperature [1-3].

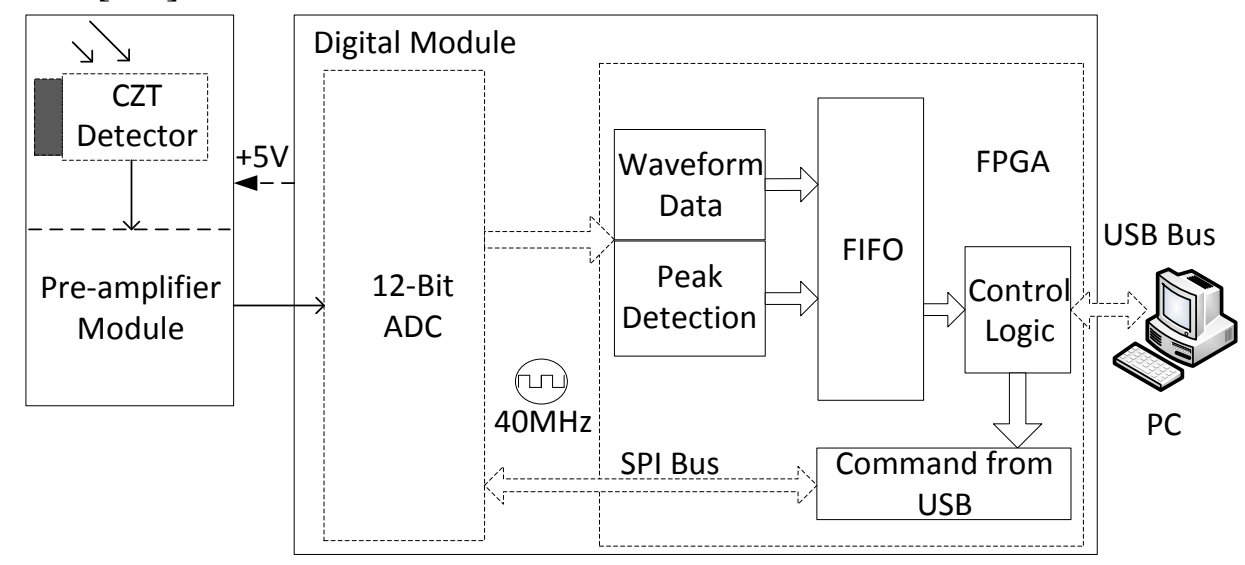

Fig. 1. Diagram of the spectroscopy. 
In this paper, a CZT detector from EURORAD with an effective thickness of $3 \mathrm{~mm}$ and a diameter of $5 \mathrm{~mm}$ is used to implement a $\gamma$ ray detector which is intended for the study of environmental monitoring. A portable low-cost, low power digital spectroscopy based on USB interface is designed. It includes a Preamplifier module and a Digital Module, as shown in Fig. 1.

The charge signal from CZT detector is collected by the preamplifier, and converted into a quasi-gauss signal which has an amplitude proportional to the charge of corresponding radiation. An ADC in the Digital Module sampled the voltage signal and the digital data are processed by the FPGA to reconstruct the waveform or seek the maximum value of the waveform. Lastly, the data is uploaded to a portable computer through the USB bus. Based on Labwindow/CVI, a graphical user interface (GUI) is developed to display the waveform of a single event or the statistical charge distribution real-timely.

\section{Hardware Design}

The spectroscopy developed is a compact, low cost, 12-bit system with 2 channels and displays the radioactive information. The main components are a preamplifier and a voltage signal digital module, which are powered by a USB port [4].

The PA, including a charge sensitive amplifier (CSA) [5] and a filter with amplifier[6], is designed by discrete devices with low cost, like AD8605, as shown in Fig. 2.The maximum output of PA can be written as:

$$
\mathrm{V}_{\mathrm{M}}=\mathrm{AQ} \text {. }
$$

In (1), A is the amplification coefficient of the preamplifier.

The digital module is designed around an IGLOO flash-based low power FPGA and ADCs which are driven by a $40 \mathrm{MHz}$ crystal oscillator, as shown in Fig.1. By adjusting the appropriate magnification, the preamplifier satisfies different requirements and converts charge into voltage signal ranged from $-1 \mathrm{~V}$ to $1 \mathrm{~V}$. The $12 \mathrm{bit}$ ADCs samples the voltage signals from the PA and the digitized data are transferred to the FPGA. The FPGA is designed to work in two mode, waveform reconstruction and peak detection, under software control. In the waveform reconstruction mode, the data from ADCs are stored after trigger. While in the peak detection mode, the mean baseline value and the maximum value of the signal are figure out firstly. Then the signal amplitude is obtained by subtracting the mean baseline value from the maximum value, which mostly eliminates the error caused by the baseline drift. Lastly, the data in FPGA are uploaded to the computer through the USB bus.

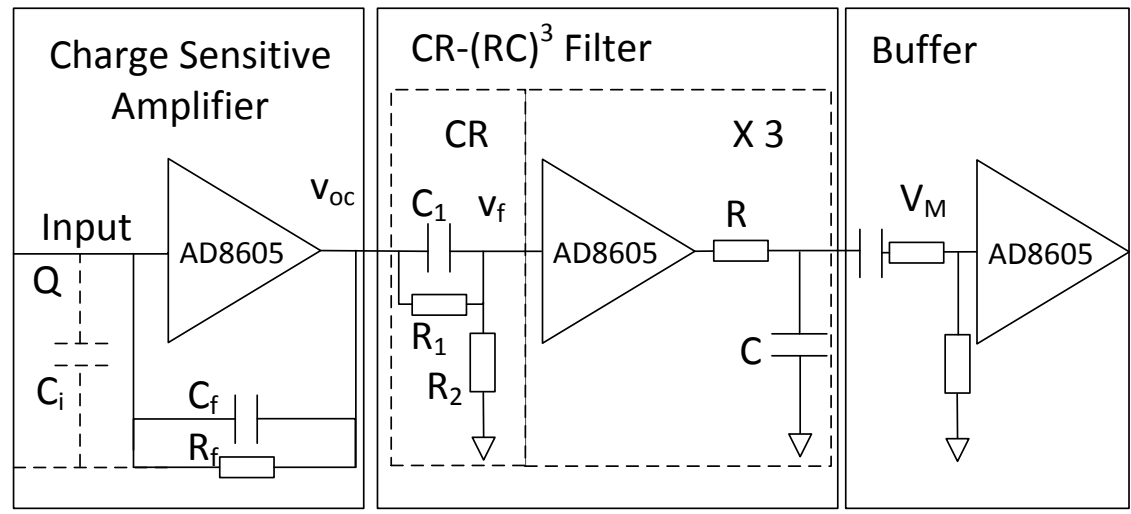

Fig. 2. Block diagram of the preamplifier.

\section{FPGA Logic and Software}

The FPGA logic, designed by a hardware description language (HDL) Verilog, realizes a function of data process and communication with the PC through a USB bus. In normal running, the FPGA 
receives commands from $\mathrm{PC}$ to configure the $\mathrm{ADC}$ and internal variables, completes the waveform reconstruction or peak detection after trigger, and transfers the data to the PC.

The control software, based on Labwindows/CVI, oversees the operation of the spectroscopy. The complete operation of the system can be understood from Fig. 3. By setting parameters of $\mathrm{T}$ and $\mathrm{M}$, the system has four modes of operation:

1) $\mathrm{T}=0, \mathrm{M}=0$ : Internal trigger and waveform reconstruction mode.

2) $\mathrm{T}=1, \mathrm{M}=0$ : External trigger and waveform reconstruction mode.

3) $\mathrm{T}=0, \mathrm{M}=1$ : Internal trigger and peak detection mode.

4) $\mathrm{T}=1, \mathrm{M}=1$ : External trigger and peak detection mode.

When the system is Power on or Reset, the system is initialized. This state ensures that the internal variables of the system are properly configured. Then commands are sent to initialize the ADC and keep it in normal running. In the internal trigger mode $(\mathrm{T}=0)$, a threshold is set and the trigger signal is generated when the input signal exceeds the threshold. Moreover, the system also can be triggered by external signal in the external trigger mode. The FPGA logic is activated by the trigger signal to begin a waveform reconstruction or peak detection determined by the mode parameter $\mathrm{M}$.

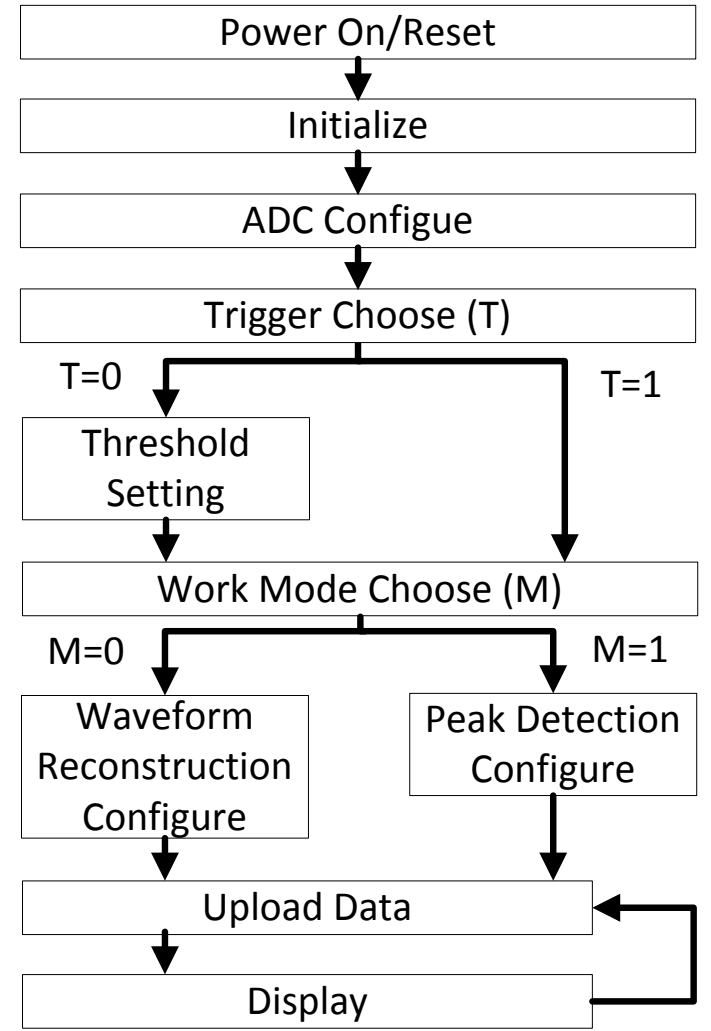

Fig. 3. The complete operation of the control software.
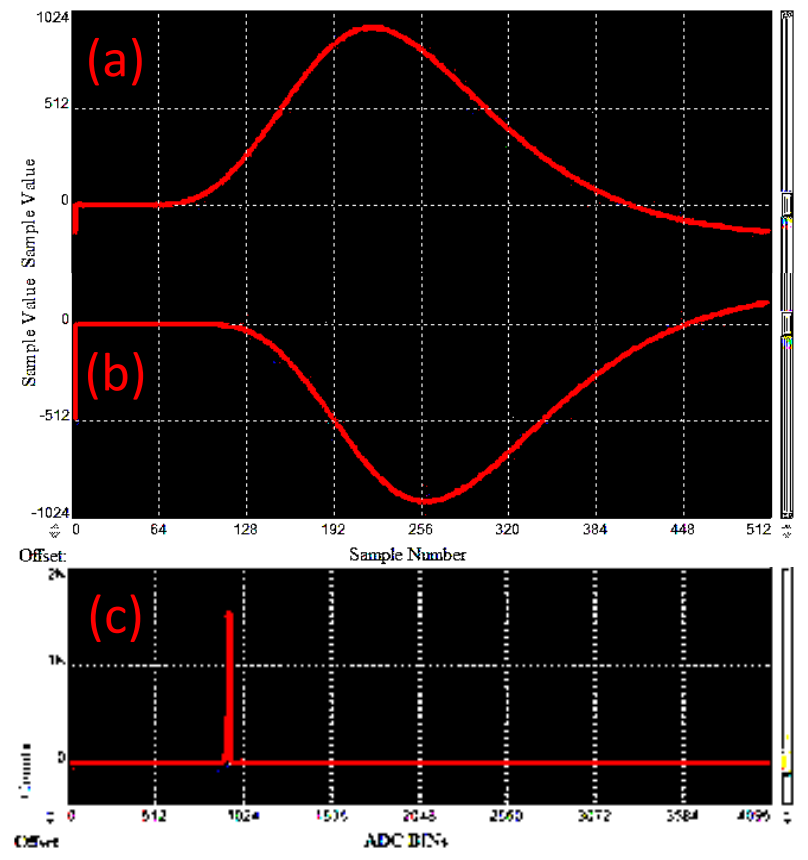

Fig. 4. The display function of the control software, (a) is the waveform of a positive charge event, (b) is the waveform of a negative charge event, and (c) is the spectrum of a large events.

The data buffered in FIFO of the FPGA are uploaded to the PC through the USB bus. Conducting with the data, the waveform is reconstructed or the statistical charge distribution of a large events is displayed in the control software. As shown in Fig. 4.

\section{Performance of the Spectroscopy}

The spectroscopy is assembled to test the performance. The equivalent noise charge (ENC) is tested without injecting charge into preamplifier and the ENC is about $0.26 \mathrm{fC}$. Then the linearity of the spectroscopy is tested. The charge from detector, which is injected into the PA, can be simulated by a pulse voltage flowing through a capacitance. A waveform generator (Tektronix, AFG3252) and an 
attenuator are adopted to generate pulse voltage signals with amplitude ranging from 0 to $24.5 \mathrm{mV}$. When the pulse voltage flows through the $10 \mathrm{pF}$ calibration capacitor, a certain amount of charge ranging from 0 to $245 \mathrm{fC}$ is injected into the PA. The linearity and resolution for negative and positive charge are shown in Fig. 5 and Fig. 6, respectively. The resolution is better than $0.3 \mathrm{fC}$.
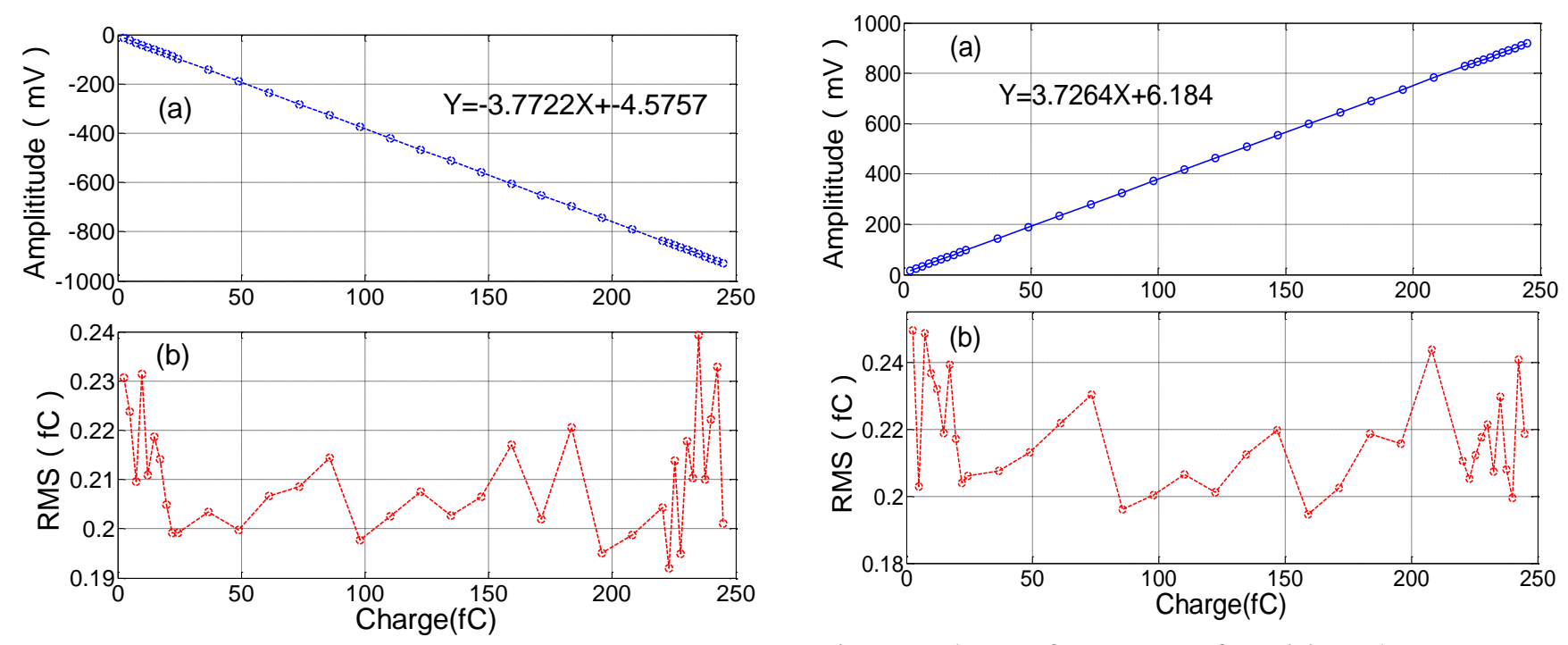

Fig. 5. The performance of negative charge measurement, (a) is linearity, (b) is RMS.

Fig. 6. The performance of positive charge measurement, (a) is linearity, (b) is RMS.

\section{Conclusion}

The portable spectroscopy, with low power consumption and low cost, has been successfully implemented. It has a simple and flexible architecture and displays the radiation information in real-time. It satisfy the CZT detector and can be extended to other applications with charge measurement demands.

\section{Acknowledgment}

This work was supported by the National Natural Science Funds of China (Grant No. 11205154).

\section{References}

[1] Takahashi T, Watanabe S. Recent progress in CdTe and CdZnTe detectors [J]. IEEE Transactions on Nuclear Science, 2001, 48(4): 950-959.

[2] Shor A, Eisen Y, Mardor I. Gamma spectroscopy with pixelated CdZnTe detectors[C]//Nuclear Science Symposium Conference Record, 2003 IEEE. IEEE, 2003, 5: 3579-3582.

[3] Zhang L, Li Y, Mao S, et al. Effects of sample processing on the performance of CdZnTe crystals[C]//Nuclear Science Symposium Conference Record, 2005 IEEE. IEEE, 2005, 5: 2976-2978.

[4] Reydarns H, Lauwereys V, Haeseldonckx D, et al. The development of a proof of concept for a smart DC/DC power plug based on USB power delivery[C]//Domestic Use of Energy (DUE), 2014 Proceedings of the Twenty-Second. IEEE, 2014: 1-4

[5] Lin M C, Syrzycki M. Current source transistor optimization methodology for noise optimized charge sensitive amplifier with fast shaper[C]//Electrical and Computer Engineering (CCECE), 2011 24th Canadian Conference on. IEEE, 2011: 000735-000738.

[6] Wulleman J. Detector-noise suppression by appropriate CR-(RC) ${ }^{\mathrm{n}}$ shaping [J]. Electronics Letters, 1996, 32(21): 1953-1954. 